\title{
Comments on supersymmetry algebra and contact term in matrix string theory
}

\section{Sanefumi Moriyama}

California Institute of Technology 452-48

Pasadena, CA 91125, U.S.A.

E-mail: moriyama@theory.caltech.edu

ABSTRACT: Following [6] relating the matrix string theory to the light-cone superstring field theory, we write down two supercharges in the matrix string theory explicitly. After checking the supersymmetry algebra at the leading order, we proceed to discuss higherorder contact terms.

Keywords: Superstrings and Heterotic Strings, M(atrix) Theories, String Field Theory 


\section{Contents}

1. Introduction 1

2. Conceptual review of light-cone superstring field theory 2

3. Matrix string theory 3

4. Supersymmetry at the leading order 5

5. Discussion on contact terms 7

A. OPE of twist fields 9

B. OPE of excited twist fields 10

G. OPE of spin fields 11

\section{Introduction}

String field theory is a standard off-shell formalism to describe fundamental string interactions in the perturbative way. Recently its importance is recognized in various contexts. Among others, the success in the PP-wave/SYM correspondence is remarkable. (See 国 for reviews and [2] for recent progress.) The dilatation matrix elements calculated in perturbative SYM theory were found to match completely with the hamiltonian matrix elements of the corresponding states in PP-wave light-cone string field theory after a basis change [3. What is surprising is that, the matching goes beyond the cubic interaction: even the contact term was found to match if we truncate the intermediate states to a particular sector [四, 5 . However, we have to confess that our understanding of the contact terms is very restricted and the meaning of this truncation still needs to be clarified. Although there is a canonical method to discuss the contact terms using the supersymmetry algebra, various questions related to its general structure remain unanswered. The main difficulty consists in its notorious complexity of the algebra.

Last summer, Dijkgraaf and Motl [6] revisited the matrix string theory [7, 8, another proposal to describe the fundamental string interaction. They related very extensively the matrix string theory to the light-cone superstring field theory in the flat space [0] and also came up with an interesting proposal on the contact term. Since on the string field theory side we have a systematic method to discuss the contact term from the supersymmetry algebra, it is very tantalizing to apply this method directly to the matrix string theory. This is our main theme in this paper. We hope we can avoid the complexity of the algebra 
on the string field theory side in this way and present a systematic calculation to prove or disprove the contact term proposal of [6]. We will see later that our method, though still has many problems, looks somewhat promising.

Another motivation for our present work is as follows. In Witten's bosonic cubic string field theory [10], besides the operator formalism [11], we have another CFT formalism [12. It should be good to have more than one description, because physical observables sometimes appear to be anomalous in the string field theory [13, 14]. We hope the matrix string theory will serve as another formalism for the light-cone superstring field theory and studying the matrix string theory will give us insights on the light-cone string field theory, and vice versa.

The contents of the rest of this paper is as follows. We start with reviewing the lightcone superstring field theory in section 2. Instead of going into the details of this theory, we only focus on the conceptual part. Especially, we explain how light-cone superstring interactions are determined from supersymmetry algebra. After that, we proceed to the matrix string theory in section 3 , explaining our strategy to the interaction terms by mimicking the light-cone superstring field theory. Following this strategy, we check the supersymmetry algebra at the first non-trivial stringy level in section 1 and proceed to discuss some problems in calculating the contact term in section 5

\section{Conceptual review of light-cone superstring field theory}

In this section we shall review the light-cone superstring field theory [9. The light-cone superstring field theory is constructed by generalizing the light-cone quantization of string theory in Green-Schwarz formalism. Hence, the start point is the covariant string worldsheet action with spacetime supersymmetries. Since we cannot quantize the action covariantly, we have to choose the light-cone gauge. After the gauge-fixing, the light-cone hamiltonian and two supercharges read

$$
\begin{aligned}
H_{0} & =\int_{0}^{2 \pi} \frac{d \sigma}{2 \pi} \frac{1}{4}\left(\left(P^{i}\right)^{2}+\left(X^{i \prime}\right)^{2}+\theta^{\alpha} \theta^{\alpha \prime}+\bar{\theta}^{\alpha} \bar{\theta}^{\alpha \prime}\right), \\
Q_{0}^{\dot{\alpha}} & =\int_{0}^{2 \pi} \frac{d \sigma}{2 \pi} \gamma_{\alpha \dot{\alpha}}^{i} \theta^{\alpha}\left(P^{i}+X^{i \prime}\right), \\
\tilde{Q}_{0}^{\dot{\alpha}} & =\int_{0}^{2 \pi} \frac{d \sigma}{2 \pi} \gamma_{\alpha \dot{\alpha}}^{i} \bar{\theta}^{\alpha}\left(P^{i}-X^{i \prime}\right),
\end{aligned}
$$

with $\theta^{\alpha}$ being the Green-Schwarz fermions. These charges satisfy the supersymmetry algebra

$$
\left\{Q_{0}^{\dot{\alpha}}, Q_{0}^{\dot{\beta}}\right\}=\left\{\tilde{Q}_{0}^{\dot{\alpha}}, \tilde{Q}_{0}^{\dot{\beta}}\right\}=2 H \delta^{\dot{\alpha} \dot{\beta}}, \quad\left[Q_{0}^{\dot{\alpha}}, H_{0}\right]=\left[\tilde{Q}_{0}^{\dot{\alpha}}, H_{0}\right]=0,
$$

with two supercharges being independent:

$$
\left\{Q_{0}^{\dot{\alpha}}, \tilde{Q}_{0}^{\dot{\beta}}\right\}=0
$$

Note that in the closed string theory we have the level-matching condition, relating the left-moving modes to right-moving modes. The algebra holds up to this constraint. 
So far we only have the free part. To construct the light-cone superstring field theory, we have to include the interaction part

$$
\begin{aligned}
H & =H_{0}+g_{s} H_{1}+g_{s}^{2} H_{2}+\cdots, \\
Q^{\dot{\alpha}} & =Q_{0}^{\dot{\alpha}}+g_{s} Q_{1}^{\dot{\alpha}}+g_{s}^{2} Q_{2}^{\dot{\alpha}}+\cdots, \\
\tilde{Q}^{\dot{\alpha}} & =\tilde{Q}_{0}^{\dot{\alpha}}+g_{s} \tilde{Q}_{1}^{\dot{\alpha}}+g_{s}^{2} \tilde{Q}_{2}^{\dot{\alpha}}+\cdots,
\end{aligned}
$$

respecting the full supersymmetry algebra order by order

$$
\left\{Q^{\dot{\alpha}}, Q^{\dot{\beta}}\right\}=\left\{\tilde{Q}^{\dot{\alpha}}, \tilde{Q}^{\dot{\beta}}\right\}=2 H \delta^{\dot{\alpha} \dot{\beta}}, \quad\left[Q^{\dot{\alpha}}, H\right]=\left[\tilde{Q}^{\dot{\alpha}}, H\right]=0 .
$$

Besides, as in the free case, we require two supercharges to be independent:

$$
\left\{Q^{\dot{\alpha}}, \tilde{Q}^{\dot{\beta}}\right\}=0 .
$$

More explicitly, if we concentrate only on one of the two supercharges, (2.5) will become

$$
\begin{aligned}
\left\{Q_{0}^{\dot{\alpha}}, Q_{0}^{\dot{\beta}}\right\} & =2 H_{0} \delta^{\dot{\alpha} \dot{\beta}}, & {\left[Q_{0}^{\dot{\alpha}}, H_{0}\right] } & =0, \\
\left\{Q_{0}^{\dot{\alpha}}, Q_{1}^{\dot{\beta}}\right\}+\left\{Q_{1}^{\dot{\alpha}}, Q_{0}^{\dot{\beta}}\right\} & =2 H_{1} \delta^{\dot{a} \dot{b}}, & {\left[Q_{0}^{\dot{\alpha}}, H_{1}\right]+\left[Q_{1}^{\dot{\alpha}}, H_{0}\right] } & =0, \\
\left\{Q_{0}^{\dot{\alpha}}, Q_{2}^{\dot{\beta}}\right\}+\left\{Q_{1}^{\dot{\alpha}}, Q_{1}^{\dot{\beta}}\right\}+\left\{Q_{2}^{\dot{\alpha}}, Q_{0}^{\dot{\beta}}\right\} & =2 H_{2} \delta^{\dot{\alpha} \dot{\beta}}, & {\left[Q_{0}^{\dot{\alpha}}, H_{2}\right]+\left[Q_{1}^{\dot{\alpha}}, H_{1}\right]+\left[Q_{2}^{\dot{\alpha}}, H_{0}\right] } & =0 .
\end{aligned}
$$

At the first non-trivial order, $Q_{1}^{\dot{\alpha}}, \tilde{Q}_{1}^{\dot{\alpha}}$ and $H_{1}$ were solved in [9]. Since these interaction vertices relate two strings Hilbert space to one string Hilbert space, it is best to represent them as ket states:

$$
\begin{aligned}
& \left|H_{1}\right\rangle_{123}=\bar{Z}^{i} Z^{j} v^{i j}(\Lambda)|V\rangle_{123} \\
& \left|Q_{1}^{\dot{\alpha}}\right\rangle_{123}=\bar{Z}^{i} s^{i \dot{\alpha}}(\Lambda)|V\rangle_{123} \\
& \left|\tilde{Q}_{1}^{\dot{\alpha}}\right\rangle_{123}=Z^{i} \tilde{s}^{i \dot{\alpha}}(\Lambda)|V\rangle_{123} .
\end{aligned}
$$

Here $|V\rangle_{123}$ represents the geometrical overlapping of two strings and one string. The quantities $Z^{i}, \bar{Z}^{i}$ and $\Lambda^{a}$ are, roughly speaking, the renormalized operators of holomorphic part of bosonic momentum $P^{i}-X^{i \prime}$, anti-holomorphic part $P^{i}+X^{i \prime}$ and fermionic momentum $\lambda$ evaluated at the interaction point respectively and $v^{i j}, s^{i \dot{\alpha}}$ and $\tilde{s}^{i \dot{\alpha}}$ are some complicate polynomials of $\Lambda$.

Apparently, there are many questions related to this construction [15]. Does this expansion end at this order? If not, how do the terms of the next order $Q_{2}^{\dot{\alpha}}, \tilde{Q}_{2}^{\dot{\alpha}}$ and $H_{2}$ look like? Can we compute the terms up to arbitrary order or do we have to calculate order by order? However, the present formalism is complicated and we do not have a satisfactory answer to these questions.

\section{Matrix string theory}

Let us turn to the matrix string theory [8. The matrix string theory proposal was motivated by compactifying [16, ] $\mathrm{M}($ atrix) theory [17] on one dimension. The definition of the matrix string theory is the low energy effective action of the two-dimensional super Yang- 
Mills theory. When the theory flows to the IR limit, it will look like a free theory of string bits propagating in the moduli space with the hamiltonian and the supercharges given by

$$
\begin{aligned}
H_{0} & =\sum_{m=1}^{N} \int_{0}^{2 \pi} \frac{d \sigma}{2 \pi} \frac{1}{4}\left(\left(P_{m}^{i}\right)^{2}+\left(X_{m}^{i \prime}\right)^{2}+\theta_{m}^{\alpha} \theta_{m}^{\alpha \prime}+\bar{\theta}_{m}^{\alpha} \bar{\theta}_{m}^{\alpha \prime}\right), \\
Q_{0}^{\dot{\alpha}} & =\sum_{m=1}^{N} \int_{0}^{2 \pi} \frac{d \sigma}{2 \pi} \gamma_{\alpha \dot{\alpha}}^{i} \theta_{m}^{\alpha}\left(P_{m}^{i}+X_{m}^{i \prime}\right), \\
\tilde{Q}_{0}^{\dot{\alpha}} & =\sum_{m=1}^{N} \int_{0}^{2 \pi} \frac{d \sigma}{2 \pi} \gamma_{\alpha \dot{\alpha}}^{i} \bar{\theta}_{m}^{\alpha}\left(P_{m}^{i}-X_{m}^{i \prime}\right) .
\end{aligned}
$$

Here the index $m$ denotes each of the string bits.

To incorporate the interaction terms, we have to return slightly from the complete IR limit and consider the least irrelevant operator. The first non-trivial order of the hamiltonian in matrix string theory is conjectured to be

$$
H_{1}=\sum_{m<n} \int_{0}^{2 \pi} \frac{d \sigma}{2 \pi}\left(\left[\tau^{i} \bar{\tau}^{j}\right] \Sigma^{i} \bar{\Sigma}^{j}\right)_{(m n)} .
$$

Here all the fields are twist fields or spin fields exchanging $m$-th and $n$-th string bits. If we take $[\sigma \bar{\sigma}](z, \bar{z})$ to be the twist field in the bosonic sector, which corresponds to the anti-periodic ground state $|0\rangle_{\mathrm{AP}},\left[\tau^{i} \bar{\tau}^{j}\right](z, \bar{z})$ is the excited twist field, corresponding to $\alpha_{-1 / 2}^{i} \bar{\alpha}_{-1 / 2}^{j}|0\rangle_{\mathrm{AP}}$. Note that the twist field $[\sigma \bar{\sigma}](z, \bar{z})$ do not decouple to the holomorphic part $\sigma(z)$ and the anti-holomorphic part $\bar{\sigma}(\bar{z})$, though we symbolically use this notation. The spin fields of the fermionic sector can be classified by $\mathbf{8}_{\mathbf{v}}$ and $\mathbf{8}_{\mathbf{c}}$, therefore we denote them as $\Sigma^{i}$ and $\Sigma^{\dot{\alpha}}$. Here we take fields with integer (half-integer) dimension to be bosons (fermions).

One evidence for this interaction term is the dimension counting. The conformal dimension of the least irrelevant operator (3.2) is $(3 / 2,3 / 2)$. This fact corresponds beautifully to the fact that the first non-trivial interaction should be proportional to the string coupling $g_{s}$. Another evidence is the idea of supersymmetry. Also, it was shown [18] that the Veneziano amplitude is reproduced from this interaction term.

Besides, in [6] the relation between the matrix string theory and the light-cone superstring field theory was made explicit. They found the quantities $v^{i j}, s^{i \dot{\alpha}}$ and $\tilde{s}^{i \dot{\alpha}}$ appearing in the first non-trivial order in the light-cone superstring field theory (2.8) are related respectively to

$$
v^{i j} \Leftrightarrow \Sigma^{i} \bar{\Sigma}^{j}, \quad s^{i \dot{\alpha}} \Leftrightarrow \Sigma^{\dot{\alpha}} \bar{\Sigma}^{i}, \quad \tilde{s}^{i \dot{\alpha}} \Leftrightarrow \Sigma^{i} \bar{\Sigma}^{\dot{\alpha}},
$$

in the matrix string theory. Therefore, comparing (3.2) with the first line of $(2.8)$, we find a complete match. Moreover, it is very plausible to write down the supercharges at the first non-trivial order as

$$
\begin{aligned}
Q_{1}^{\dot{\alpha}} & =-\sqrt{2} \sum_{m<n} \int_{0}^{2 \pi} \frac{d \sigma}{2 \pi}\left(\left[\sigma \bar{\tau}^{i}\right] \Sigma^{\dot{\alpha}} \bar{\Sigma}^{i}\right)_{(m n)}, \\
\tilde{Q}_{1}^{\dot{\alpha}} & =-\sqrt{2} \sum_{m<n} \int_{0}^{2 \pi} \frac{d \sigma}{2 \pi}\left(\left[\tau^{i} \bar{\sigma}\right] \Sigma^{i} \bar{\Sigma}^{\dot{\alpha}}\right)_{(m n)},
\end{aligned}
$$

where $\left[\tau^{i} \bar{\sigma}\right](z, \bar{z})$ and $\left[\sigma \bar{\tau}^{i}\right](z, \bar{z})$ correspond respectively to $\alpha_{-1 / 2}^{i}|0\rangle_{\mathrm{AP}}$ and $\bar{\alpha}_{-1 / 2}^{i}|0\rangle_{\mathrm{AP}}$. 
Note that, we can rewrite the integral of $\sigma$ in terms of the holomorphic coordinate $z$ or the anti-holomorphic one $\bar{z}$. Although for a conserved charge

$$
Q=\int_{C} d z j+d \bar{z} \bar{j}
$$

$j$ and $\bar{j}$ must satisfy

$$
\bar{\partial} j-\partial \bar{j}=0,
$$

we have not succeeded in separating the expressions of (3.2) or (3.4) into $j$ and $\bar{j}$. So, in (3.2) and (3.4) the integral contour is considered to be strictly the circle centered at the origin.

It is very important to work out the supersymmetry algebra, because the supersymmetry algebra is the main guiding principle in constructing the light-cone superstring field theory. As reviewed in the previous section, to investigate the contact terms, we have to calculate (2.7) in higher orders. For that purpose we start with the leading order. In the following sections, we shall elaborate the supersymmetry algebra at the first non-trivial order and then proceed to the contact terms.

\section{Supersymmetry at the leading order}

In this section, we shall check the supersymmetry algebra at the leading order. Note that, conceptually we have already known the result, because at this order the supersymmetry algebra was worked out in the light-cone superstring field theory by [9, and its relation to the matrix string theory was explored by [6]. Technically, the supersymmetry algebra was mostly worked out in the original paper [B], except a few details about including both the holomorphic and anti-holomorphic parts and about confirming the independence between two supercharges (2.6). However, we believe that it is important to make the leading order supersymmetry algebra clear at this point.

Let us begin with the free part. We would like to show the first line of (2.7). First of all, we have to rewrite the expressions (3.1) in terms of the holomorphic coordinates:

$$
\begin{aligned}
H_{0} & =-\frac{1}{2} \int \frac{d z}{2 \pi} z\left(\left(\partial X^{i}\right)^{2}+\theta^{\alpha} \partial \theta^{\alpha}\right) \\
Q_{0}^{\dot{\alpha}} & =\int \frac{d z}{2 \pi} \sqrt{i z} \gamma_{\alpha \dot{\alpha}}^{i} \theta^{\alpha} \partial X^{i} .
\end{aligned}
$$

Here we drop the indices for the string bit ( $m$ in (3.1)). Since there is no interaction between string bits in the free case, we pick up a particular string bit and sum them up after the calculation. Also, the hamiltonian can be written in terms of either the holomorphic variable or the anti-holomorphic one because we always restrict our Hilbert space to the subspace satisfying the level-matching condition, but we shall choose the holomorphic one here.

In the calculation we need the OPE of $\partial X^{i}$ and $\theta^{a}$ between themselves. Since they are free fields, the OPE are just

$$
\begin{aligned}
\partial X^{i}(z) \cdot \partial X^{j}(0) & \sim-\frac{\delta^{i j}}{z^{2}}+: \partial X^{i} \partial X^{j}:(0)+O(z), \\
\theta^{\alpha}(z) \cdot \theta^{\beta}(0) & \sim \frac{\delta^{\alpha \beta}}{z}+: \theta^{\alpha} \theta^{\beta}:(0)+O(z) .
\end{aligned}
$$


Using these OPE we can calculate the commutation relation by the usual trick of deforming the contours at the same radius into a small one encircling the singularity. The relation $\left\{Q_{0}^{\dot{\alpha}}, Q_{0}^{\dot{\beta}}\right\}=2 H_{0} \delta^{\dot{\alpha} \dot{\beta}}$ is fairly easy to show, though in calculating $\left[Q_{0}^{\dot{\alpha}}, H_{0}\right]$ we find

$$
\left[Q_{0}^{\dot{\alpha}}, H_{0}\right]=\int_{0} \frac{d w}{2 \pi} \int_{w} \frac{d z}{2 \pi} \sqrt{i z}(-w)\left(-3 \frac{\gamma_{\alpha \dot{\alpha}}^{i} \theta^{\alpha} \partial X^{i}(w)}{(z-w)^{2}}-\frac{\partial\left(\gamma_{\alpha \dot{\alpha}}^{i} \theta^{\alpha} \partial X^{i}\right)(w)}{z-w}\right) .
$$

After expanding $\sqrt{z}$ and picking up the residue, we have to integrate by parts in the second term to show both terms cancel identically.

Relations for the other supercharge $\tilde{Q}_{0}^{\dot{\alpha}}$ can be found in the same way. Since holomorphic fields and anti-holomorphic fields do not have non-trivial OPE, we also find our choice for two supercharges is good: they anti-commute with each other, $\left\{Q_{0}^{\dot{\alpha}}, \tilde{Q}_{0}^{\dot{\beta}}\right\}=0$.

Now let us proceed to the first non-trivial order. For this purpose, let us rewrite (3.2) and (3.4) as

$$
\begin{aligned}
H_{1} & =\int \frac{d z}{2 \pi} \sqrt{i z} \overline{i z}^{3 / 2}\left(\left[\tau^{i} \bar{\tau}^{j}\right] \Sigma^{i} \bar{\Sigma}^{j}\right), \\
Q_{1}^{\dot{\alpha}} & =-\sqrt{2} \int \frac{d z}{2 \pi} \overline{i z}^{3 / 2}\left(\left[\sigma \bar{\tau}^{j}\right] \Sigma^{\dot{\alpha}} \bar{\Sigma}^{j}\right) .
\end{aligned}
$$

Here we drop the string bit indices $(m n)$ again. We shall fix one particular pair $(m n)$ of the string bits in the first order expression (4.4) and rewrite the quadratic terms of this pair in (4.1) into sum and difference:

$$
\mathcal{O}_{m} \mathcal{P}_{m}+\mathcal{O}_{n} \mathcal{P}_{n}=\frac{\mathcal{O}_{m}+\mathcal{O}_{n}}{\sqrt{2}} \frac{\mathcal{P}_{m}+\mathcal{P}_{n}}{\sqrt{2}}+\frac{\mathcal{O}_{m}-\mathcal{O}_{n}}{\sqrt{2}} \frac{\mathcal{P}_{m}-\mathcal{P}_{n}}{\sqrt{2}}
$$

with $\mathcal{O}$ and $\mathcal{P}$ being $X^{i}$ or $\theta^{\alpha}$. Since the first order expression (4.4) with indices $(m n)$ exchanges two string bits $m$ and $n$, it have non-trivial OPE only with the difference term in (4.5). After this calculation we sum up over our choice $(m n)$. In other words, our expression at the zeroth order (4.1) has the implicit indices $(m n)$ now, meaning each operator is the difference of that with index $m$ and that with $n$.

Now we need some non-trivial OPE. These OPE are evaluated in our appendices.

$$
\begin{aligned}
\partial X^{i}(z) \cdot \sigma(0) & \sim \frac{\eta^{*}}{\sqrt{z}}\left(\tau^{i}(0)+O(z)\right), \\
\partial X^{i}(z) \cdot \tau^{j}(0) & \sim \frac{\eta^{*}}{\sqrt{z}}\left(\frac{\delta^{i j}}{2 z} \sigma(0)+\frac{\delta^{i j}}{4} \partial \sigma(0)+\left(\tau^{i j}-\frac{\delta^{i j}}{4} \partial \sigma\right)(0)+O(z)\right), \\
\theta^{\alpha}(z) \cdot \Sigma^{\dot{\alpha}}(0) & \sim \frac{\eta^{*}}{\sqrt{2 z}}\left(\gamma_{\alpha \dot{\alpha}}^{i} \Sigma^{i}(0)+O(z)\right), \\
\theta^{\alpha}(z) \cdot \Sigma^{i}(0) & \sim \frac{\eta^{*}}{\sqrt{2 z}}\left(\gamma_{\alpha \dot{\alpha}}^{i} \Sigma^{\dot{\alpha}}(0)+\frac{z}{2} \gamma_{\alpha \dot{\alpha}}^{i} \partial \Sigma^{\dot{\alpha}}(0)+\langle ?\rangle z u_{\dot{\alpha} \dot{\beta} \dot{\gamma}}^{i \alpha} \Sigma^{\dot{\alpha}} \Sigma^{\dot{\beta}} \Sigma^{\dot{\gamma}}(0)+O\left(z^{2}\right)\right),
\end{aligned}
$$

with $\eta^{*}=\exp (-i \pi / 4)$ and $u_{\dot{\alpha} \dot{\beta} \dot{\gamma}}^{i \alpha}=-\gamma_{[\dot{\alpha} \dot{\beta}}^{i j} \gamma_{\dot{\gamma}]}^{j}$. As we noted previously, the twist fields do not decouple between the holomorphic parts and the anti-holomorphic parts in general. We write down the first two formulas in this way because they hold independently of excitations by anti-holomorphic bosons. Also, $\langle ?\rangle$ in the third relation means a coefficient which is 
not fixed. Fortunately, this is not necessary in our calculation. The doubly excited twist field $\tau^{i j}$ relates to $\alpha_{-1 / 2}^{i} \alpha_{-1 / 2}^{j}|0\rangle_{\mathrm{AP}}$ and the expression in the parenthesis $\left(\tau^{i j}-\delta^{i j} \partial \sigma / 4\right)$ denotes the traceless combination, which has no singularity in OPE with $\sigma$ itself. Also, the twist field and the spin field are primary fields, so we have

$$
\begin{aligned}
T_{b}(z) \cdot \sigma(0) & \sim \frac{1}{2 z^{2}} \sigma(0)+\frac{1}{z} \partial \sigma(0)+O\left(z^{0}\right), \\
T_{f}(z) \cdot \Sigma^{\dot{\alpha}}(0) & \sim \frac{1}{2 z^{2}} \Sigma^{\dot{\alpha}}(0)+\frac{1}{z} \partial \Sigma^{\dot{\alpha}}(0)+O\left(z^{0}\right),
\end{aligned}
$$

with $T_{b}$ and $T_{f}$ being the boson part and fermion part of the energy momentum tensor, respectively:

$$
T_{b}=-\frac{1}{2}: \partial X^{i} \partial X^{i}:, \quad T_{f}=-\frac{1}{2}: \theta^{\alpha} \partial \theta^{\alpha}: .
$$

Using all these OPE, a proof of supersymmetry algebra is possible. With the help of following two formulas,

$$
\begin{aligned}
{\left[Q_{0}^{\dot{\alpha}}, \sigma \Sigma^{\dot{\beta}}(w)\right] } & =-\frac{\sqrt{i w}}{\sqrt{2}} \gamma_{\alpha \dot{\alpha}}^{i} \gamma_{\alpha \dot{\beta}}^{j} \tau^{i} \Sigma^{j}(w), \\
\left\{Q_{0}^{\dot{\alpha}}, \tau^{i} \Sigma^{i}(w)\right\} & =-\frac{\sqrt{2 i}}{\sqrt{w}}\left(\sigma \Sigma^{\dot{\alpha}}(w)+w \partial\left(\sigma \Sigma^{\dot{\alpha}}\right)(w)\right),
\end{aligned}
$$

we can prove the first non-trivial order of the supersymmetry algebra, the second line of (2.7),

$$
\left\{Q_{0}^{\dot{\alpha}}, Q_{1}^{\dot{\beta}}\right\}+\left\{Q_{1}^{\dot{\alpha}}, Q_{0}^{\dot{\beta}}\right\}=2 H_{1} \delta^{\dot{a} \dot{b}}, \quad\left[Q_{0}^{\dot{\alpha}}, H_{1}\right]+\left[Q_{1}^{\dot{\alpha}}, H_{0}\right]=0
$$

as well as independence between two supercharges:

$$
\left\{Q_{0}^{\dot{\alpha}}, \tilde{Q}_{1}^{\dot{\beta}}\right\}+\left\{Q_{1}^{\dot{\alpha}}, \tilde{Q}_{0}^{\dot{\beta}}\right\}=0 .
$$

\section{Discussion on contact terms}

Now we have proved the supersymmetry algebra at the first non-trivial order. Let us proceed to discuss the contact terms in the systematic method as summarized in section 2.

As is obvious by now, our strategy to the contact terms is to calculate $\left\{Q_{1}^{\dot{\alpha}}, Q_{1}^{\dot{\beta}}\right\}$ in (2.7) and try to determine the next order $Q_{2}^{\dot{\alpha}}$ and $H_{2}$. Here we have not succeeded in this goal. We only discuss some problems of this calculation.

We have three types of terms for $\left\{Q_{1}^{\dot{\alpha}}, Q_{1}^{\dot{\beta}}\right\}$, depending on relative interaction points: $\left\{Q_{1(k l)}^{\dot{\alpha}}, Q_{1(k l)}^{\dot{\beta}}\right\},\left\{Q_{1(k l)}^{\dot{\alpha}}, Q_{1(l m)}^{\dot{\beta}}\right\}$, and $\left\{Q_{1(k l)}^{\dot{\alpha}}, Q_{1(m n)}^{\dot{\beta}}\right\}$. As discussed in [6], on the SYM side of the PP-wave/SYM correspondence the Feynman diagram looks like the second type. This leads them to make a proposal for the contact term and investigate the $Z_{3}$ twist field carefully. However, in order to match with the SYM calculation, on the lightcone superstring field theory side [4, 5] we also have to consider the contribution somewhat similar to the first type. This type of terms corresponds to the interaction where one string splits into two and these two strings joins into one again at the same interaction point. The third type is trivial. Since two operators in the OPE of $Q_{1}$ relate to different string bits, there is no singularity appearing in the OPE. Therefore, this type of contact term should be vanishing. 
Let us concentrate on the first type $\left\{Q_{1(k l)}^{\dot{\alpha}}, Q_{1(k l)}^{\dot{\beta}}\right\}$. Note that since our hamiltonian and supercharges, (3.2) and (3.4), are not written in the expression of conserved charges (3.5), we cannot deform the integral contour arbitrarily. However, we are considering the commutation relation at the same radius. Hence, apart from the contribution where two operators are close to each other, two integral contours in the commutation relation should cancel each other. Therefore, we are allowed to consider the OPE of these operators. The leading OPE are read off from the dimensions:

$$
\begin{aligned}
{\left[\sigma \bar{\tau}^{i}\right](z, \bar{z}) \cdot\left[\sigma \bar{\tau}^{j}\right](0,0) } & \sim \frac{\delta^{i j}}{2 z \bar{z}^{2}}+O\left(z^{0}\right), \\
\Sigma^{\dot{\alpha}}(z) \cdot \Sigma^{\dot{\beta}}(0) & \sim \frac{\delta^{\dot{\alpha} \dot{\beta}}}{z}+O\left(z^{0}\right), \\
\bar{\Sigma}^{i}(\bar{z}) \cdot \bar{\Sigma}^{j}(0) & \sim \frac{\delta^{i j}}{\bar{z}}+O\left(\bar{z}^{0}\right) .
\end{aligned}
$$

The subleading orders are also relevant after multiplying the twist fields and the spin fields.

We would like to integrate these OPE over $z$ and $w$ and find exactly the commutator $\left\{Q_{1(k l)}^{\dot{\alpha}}, Q_{1(k l)}^{\dot{\beta}}\right\}$. However, there are some problems. First of all, as we have noted, we cannot deform the contour arbitrarily. Therefore, the commutators at the same radius will depend on the regularization. At present we do not have a good principle to choose the regularization. A natural one may be to encircle the singularity after cancelling the contribution where two operators are separated far away, as the usual contour deformation technique. If we adopt this regularization, the second problem appears. The integration of the most singular term

$$
\int_{0} \frac{d w}{2 \pi i} \int_{w} \frac{d z}{2 \pi i} \bar{z}^{3 / 2} \bar{w}^{3 / 2} \frac{1}{(z-w)^{2}(\bar{z}-\bar{w})^{3}}
$$

gives divergent contribution. We need an interpretation for it.

This divergence in $\left\{\left(Q_{1}\right)_{(k l)},\left(Q_{1}\right)_{(k l)}\right\}$ may not be surprising. We can perform a similar calculation in the PP-wave string field theory. When we match two geometrical interaction vertices $|V\rangle$, we need the formula:

$$
\exp \left(\frac{1}{2} a N a\right) \exp \left(\frac{1}{2} a^{\dagger} M a^{\dagger}\right)|0\rangle=\frac{1}{\sqrt{\operatorname{det}(1-M N)}} \exp \left(\frac{1}{2} a^{\dagger} M \frac{1}{1-N M} a^{\dagger}\right)|0\rangle .
$$

However, if we take the mass parameter $\mu$ of the PP-wave background to infinity, these matrices $M, N$ are the same orthonormal matrices. Therefore, it looks singular in this limit.

The situation for the second type $\left\{\left(Q_{1}\right)_{(k l)},\left(Q_{1}\right)_{(l m)}\right\}$ is more complicated. Even thought it is easy to guess the OPE between the twist fields $[\sigma \bar{\sigma}]_{(k l)}$ and $[\sigma \bar{\sigma}]_{(l m)}$ from the multiplication of the permutation group $(k l)(l m)=(k l m)$ and the dimension counting, it is not so easy to calculate the OPE of the excited twist fields $\left[\sigma \bar{\tau}^{i}\right]_{(k l)}(z, \bar{z}) \cdot\left[\sigma \bar{\tau}^{j}\right]_{(l m)}(0,0)$ because they are excited by different fields. 


\section{Acknowledgments}

We would like to thank Jaume Gomis, Lubos Motl, Hirosi Ooguri, John Schwarz and especially Takuya Okuda for useful discussions. We are also grateful to Yuji Okawa for pointing out a mistake in the previous version of this paper. This research was supported in part by the DOE grant DE-FG03-92-ER40701.

\section{A. OPE of twist fields}

In this appendix, we shall show the first line of (4.6). We begin with showing

$$
\partial X^{i}(z) \cdot[\sigma \bar{\sigma}](0,0) \sim \frac{\eta^{*}}{\sqrt{z}}\left(\left[\tau^{i} \bar{\sigma}\right](0,0)+O(z)\right)
$$

with no excitations by the anti-holomorphic bosons. For this purpose let us consider the four point function $\left\langle[\sigma \bar{\sigma}]\left(z_{1}, \bar{z}_{1}\right) \partial X^{i}\left(z_{2}\right) \partial X^{j}\left(z_{3}\right)[\sigma \bar{\sigma}]\left(z_{4}, \bar{z}_{4}\right)\right\rangle$. By taking two bosons $\partial X^{i}(z)$ close to two twist fields $[\sigma \bar{\sigma}](z, \bar{z})$, respectively, we can read off the above OPE.

The calculation of the four point functions $\left\langle[\sigma \bar{\sigma}]\left(z_{1}, \bar{z}_{1}\right) \partial X^{i}\left(z_{2}\right) \partial X^{j}\left(z_{3}\right)[\sigma \bar{\sigma}]\left(z_{4}, \bar{z}_{4}\right)\right\rangle$ is standard and can be found, for example in the textbook [19]. Since it will also be necessary in the next appendix we shall recapitulate it briefly in the following.

Using the OPE of bosons (4.2) and the mode expansion of bosons in the twisted sector

$$
i \partial X^{i}(z)=\sum_{r \in Z+1 / 2} \frac{\alpha_{r}}{z^{r+1}},
$$

we find the commutation relation is unchanged as in the untwisted sector

$$
\left[\alpha_{r}^{i}, \alpha_{s}^{j}\right]=r \delta^{i j} \delta_{r+s}
$$

except that the indices run over half-integers now. Hence, we can compute the two point function in the twisted sector

$$
\operatorname{AP}\left\langle 0\left|\partial X^{i}\left(z_{2}\right) \partial X^{j}\left(z_{3}\right)\right| 0\right\rangle_{\mathrm{AP}}=-\frac{\sqrt{z_{2} / z_{3}}+\sqrt{z_{3} / z_{2}}}{2\left(z_{2}-z_{3}\right)^{2}} \delta^{i j},
$$

where $|0\rangle_{\mathrm{AP}}$ denotes the anti-periodic twisted ground state $[\sigma \bar{\sigma}](0,0)|0\rangle$. In other words, this quantity can be interpreted as the four point function,

$$
\lim _{z_{1} \rightarrow \infty, z_{4} \rightarrow 0} z_{1} \bar{z}_{1}\left\langle[\sigma \bar{\sigma}]\left(z_{1}, \bar{z}_{1}\right) \partial X^{i}\left(z_{2}\right) \partial X^{j}\left(z_{3}\right)[\sigma \bar{\sigma}]\left(z_{4}, \bar{z}_{4}\right)\right\rangle .
$$

Restoring the dependence on $z_{1}$ and $z_{4}$ from the general argument of four point functions in two dimensional CFT, we can rewrite the previous results in a more democratic way:

$$
\left\langle[\sigma \bar{\sigma}]\left(z_{1}, \bar{z}_{1}\right) \partial X^{i}\left(z_{2}\right) \partial X^{j}\left(z_{3}\right)[\sigma \bar{\sigma}]\left(z_{4}, \bar{z}_{4}\right)\right\rangle=-\frac{1}{z_{14} z_{23}^{2} \bar{z}_{14}} \sqrt{\frac{z_{13} z_{24}}{z_{12} z_{34}}}\left(1-\frac{z_{14} z_{23}}{2 z_{13} z_{24}}\right) \delta^{i j}
$$

with $z_{i j} \equiv z_{i}-z_{j}$. 
Now let us take the limit $\left|z_{12}\right|,\left|z_{34}\right| \ll\left|z_{14}\right|$ in A.6. .

$$
\left\langle[\sigma \bar{\sigma}]\left(z_{1}, \bar{z}_{1}\right) \partial X^{i}\left(z_{2}\right) \partial X^{j}\left(z_{3}\right)[\sigma \bar{\sigma}]\left(z_{4}, \bar{z}_{4}\right)\right\rangle \simeq-\frac{\delta^{i j}}{2 z_{14}^{2} \bar{z}_{14} \sqrt{z_{12} z_{34}}} .
$$

This means that if we choose the excited twist field $\left[\tau^{i} \bar{\sigma}\right](z, \bar{z})$ to correspond to the state $\alpha_{-1 / 2}^{i}|0\rangle_{\mathrm{AP}}$, then we have the OPE (A.1) and

$$
\left[\tau^{i} \bar{\sigma}\right](z, \bar{z}) \cdot\left[\tau^{j} \bar{\sigma}\right](0,0) \sim \frac{\delta^{i j}}{2 z^{2} \bar{z}}+O\left(z^{-1}\right)
$$

As is obvious by repeating the same calculation, this calculation does not depend on the excitations by anti-holomorphic bosons $\bar{\alpha}_{r}^{i}$. Therefore, we can rewrite the previous result symbolically as

$$
\partial X^{i}(z) \cdot \sigma(0) \sim \frac{\eta^{*}}{\sqrt{z}}\left(\tau^{i}(0)+O(z)\right) .
$$

The first line in (4.6) is shown in this way.

\section{B. OPE of excited twist fields}

This appendix is devoted to the second line of (1.6). As in the previous appendix, this calculation also does not depend on the excitations by anti-holomorphic bosons $\bar{\alpha}_{r}^{i}$. Therefore we drop the anti-holomorphic dependence from the beginning. Note again that this is purely symbolic notation. In other computations the holomorphic part and the antiholomorphic part do not decouple in general.

Since the excited twist field $\tau^{i}(z)$ corresponds to $\alpha_{-1 / 2}^{i}|0\rangle_{\mathrm{AP}}$, the four point function

$$
\lim _{z_{1} \rightarrow \infty, z_{4} \rightarrow 0} z_{1}^{2}\left\langle\tau^{i}\left(z_{1}\right) \partial X^{k}\left(z_{2}\right) \partial X^{l}\left(z_{3}\right) \tau^{j}\left(z_{4}\right)\right\rangle
$$

can be calculated as

$$
\begin{aligned}
& { }_{\operatorname{AP}}\left\langle 0\left|\alpha_{1 / 2}^{i} \partial X^{k}\left(z_{2}\right) \partial X^{l}\left(z_{3}\right) \alpha_{-1 / 2}^{j}\right| 0\right\rangle_{\mathrm{AP}}= \\
& \quad=-\frac{1}{4} \frac{\sqrt{z_{2} / z_{3}}+\sqrt{z_{3} / z_{2}}}{\left(z_{2}-z_{3}\right)^{2}} \delta^{i j} \delta^{k l}-\frac{1}{4} \frac{\sqrt{z_{2} / z_{3}}}{z_{2} z_{3}} \delta^{i k} \delta^{j l}-\frac{1}{4} \frac{\sqrt{z_{3} / z_{2}}}{z_{2} z_{3}} \delta^{i l} \delta^{j k} .
\end{aligned}
$$

Again from the general arguments of four point functions, we can rewrite it into

$$
\begin{aligned}
& \left\langle\tau^{i}\left(z_{1}\right) \partial X^{k}\left(z_{2}\right) \partial X^{l}\left(z_{3}\right) \tau^{j}\left(z_{4}\right)\right\rangle= \\
& \quad=-\frac{1}{4} \sqrt{\frac{z_{13} z_{24}}{z_{12} z_{34}}}\left\{\left(1+\frac{z_{12} z_{34}}{z_{13} z_{24}}\right) \frac{\delta^{i j} \delta^{k l}}{\left(z_{14} z_{23}\right)^{2}}+\frac{\delta^{i k} \delta^{j l}}{z_{12} z_{34} z_{13} z_{24}}+\frac{\delta^{i l} \delta^{j k}}{\left(z_{13} z_{24}\right)^{2}}\right\} .
\end{aligned}
$$

As in the previous appendix, we consider the OPE of $\partial X^{i}$ and $\tau^{j}$. Note that, from the Lorentz symmetry and dimension analysis, terms that we can have in the operator expansion should be restricted to $\delta^{i j} \sigma / z^{3 / 2}, \delta^{i j} \partial \sigma / z^{1 / 2}$ and $\left(\tau^{i j}-\partial \sigma / 4\right) / z^{1 / 2}$ up to $O\left(z^{1 / 2}\right)$. Here $\tau^{i j}$ corresponds to $\alpha_{-1 / 2}^{i} \alpha_{-1 / 2}^{j}|0\rangle_{\mathrm{AP}}$ and the subtraction in the last term comes from the traceless condition. Note that the derivative $\partial \sigma$ is the descendent of the twist field 
$L_{-1} \sigma$, hence this field corresponds to $(1 / 2) \alpha_{-1 / 2}^{i} \alpha_{-1 / 2}^{i}|0\rangle_{\mathrm{AP}}$. These correspondence implies the OPE

$$
\begin{gathered}
\partial \sigma(z) \cdot\left(\tau^{i j}-\frac{\delta^{i j}}{4} \partial \sigma\right)(0) \sim 0+O\left(z^{-4}\right) \\
\left(\tau^{i k}-\frac{\delta^{i k}}{4} \partial \sigma\right)(z) \cdot\left(\tau^{j l}-\frac{\delta^{j l}}{4} \partial \sigma\right)(0) \\
\sim \frac{\delta^{i k} \delta^{j l}-4\left(\delta^{i j} \delta^{k l}+\delta^{i l} \delta^{j k}\right)}{16 z^{3}}+O\left(z^{-4}\right) .
\end{gathered}
$$

In order to determine the coefficients, let us take the limit $\left|z_{12}\right|,\left|z_{34}\right| \ll\left|z_{14}\right|$ again in (B.3):

$$
\begin{aligned}
\left\langle\tau^{i}\left(z_{1}\right) \partial X^{k}\left(z_{2}\right) \partial X^{l}\left(z_{3}\right) \tau^{j}\left(z_{4}\right)\right\rangle \simeq & -\frac{1}{4 z_{14}\left(z_{12} z_{34}\right)^{3 / 2}} \times \\
& \times\left(\delta^{i k} \delta^{j l}\left(1+\frac{1}{2} \frac{z_{12}}{z_{14}}+\frac{1}{2} \frac{z_{34}}{z_{14}}+\frac{1}{4} \frac{z_{12} z_{34}}{z_{14}^{2}}\right)+\right. \\
& \left.+\delta^{i j} \delta^{k l} \frac{z_{12} z_{34}}{z_{14}^{2}}+\delta^{i l} \delta^{j k} \frac{z_{12} z_{34}}{z_{14}^{2}}\right)
\end{aligned}
$$

and read off the coefficients from this expansion. The result is

$$
\partial X^{i}(z) \cdot \tau^{j}(0) \sim \frac{\eta^{*}}{\sqrt{z}}\left(\frac{\delta^{i j}}{2 z} \sigma(0)+\frac{\delta^{i j}}{4} \partial \sigma(0)+\left(\tau^{i j}-\frac{\delta^{i j}}{4} \partial \sigma\right)(0)+O(z)\right) .
$$

\section{OPE of spin fields}

In this appendix, we shall calculate the OPE of spin fields, the last one in (4.6),

$$
\theta^{\alpha}(z) \cdot \Sigma^{i}(-z) \sim \frac{\eta^{*}}{\sqrt{4 z}}\left(\gamma_{\alpha \dot{\alpha}}^{i} \Sigma^{\dot{\alpha}}(0)+\langle ?\rangle z u_{\dot{\alpha} \dot{\beta} \dot{\gamma}}^{i \alpha}: \Sigma^{\dot{\alpha}} \Sigma^{\dot{\beta}} \Sigma^{\dot{\gamma}}:(0)+O\left(z^{2}\right)\right) .
$$

The method adopted in this appendix is completely different from the previous two appendices. Since we can represent the spin fields by free bosons, our strategy is first to restrict terms which can appear in the expansion and then identify the coefficients by calculating the OPE of some typical terms with the free field realization.

Let us consider $\gamma_{\alpha \dot{\alpha}}^{i} \theta^{\alpha}(z) \cdot \Sigma^{i}(-z)$ for the evaluation of (C.1). Since only terms having the same Lorentz indices and correct conformal dimensions can appear in the expansion, the possible terms up to $O\left(z^{3 / 2}\right)$ are restricted to

$$
\frac{1}{\sqrt{z}} \Sigma^{\dot{\alpha}}, \quad \sqrt{z} \partial \Sigma^{\dot{\alpha}}, \quad \sqrt{z} t_{\dot{\alpha} \dot{\beta} \dot{\gamma} \dot{\delta}}^{i i} \Sigma^{\dot{\beta}} \Sigma^{\dot{\gamma}} \Sigma^{\dot{\delta}},
$$

with $t_{\dot{\alpha} \dot{\beta} \dot{\gamma} \dot{\delta}}^{i j}$ defined by

$$
t_{\dot{\alpha} \dot{\beta} \dot{\gamma} \dot{\delta}}^{i j}=\gamma_{[\dot{\alpha} \dot{\beta}}^{i k} \gamma_{\dot{\gamma} \dot{\delta}]}^{j k}
$$

Since $t_{\dot{\alpha} \dot{\beta} \dot{\gamma} \dot{\delta}}^{i j}$ is traceless, the last term vanishes identically. 
We have only to determine the coefficient of each term by the free field realization. If we combine the real fermions into complex ones as

$$
\begin{array}{rlrl}
\Theta^{A} & \equiv \frac{1}{\sqrt{2}}\left(\theta^{A}+i \theta^{A+4}\right), & \Theta^{\bar{A}} & \equiv \frac{1}{\sqrt{2}}\left(\theta^{A}-i \theta^{A+4}\right), \\
\mathcal{S}^{A} & \equiv \frac{1}{\sqrt{2}}\left(\Sigma^{2 A-1}+i \Sigma^{2 A}\right), & \mathcal{S}^{\bar{A}} & \equiv \frac{1}{\sqrt{2}}\left(\Sigma^{2 A-1}-i \Sigma^{2 A}\right), \\
\mathcal{S}^{\dot{A}} & \equiv \frac{1}{\sqrt{2}}\left(\Sigma^{\dot{A}}+i \Sigma^{\dot{A}+4}\right), & \mathcal{S}^{\dot{\bar{A}}} \equiv \frac{1}{\sqrt{2}}\left(\Sigma^{\dot{A}}-i \Sigma^{\dot{A}+4}\right),
\end{array}
$$

these complex fermions are realized in terms of free bosons $\phi^{A}(A=1, \ldots, 4)$ by,

$\Theta^{A}=e^{i q_{B}^{A} \phi^{B}}, \Theta^{\bar{A}}=e^{-i q_{B}^{A} \phi^{B}}, \quad \mathcal{S}^{A}=e^{i \phi^{A}}, \mathcal{S}^{\bar{A}}=e^{-i \phi^{A}}, \quad \mathcal{S}^{\dot{A}}=e^{i q_{B}^{\dot{A}} \phi^{B}}, \quad \mathcal{S}^{\dot{\bar{A}}}=e^{-i q_{B}^{\dot{A}} \phi^{B}}$,

with

$$
\begin{aligned}
& q^{1}=\frac{1}{2}(1,1,1,1), \quad q^{2}=\frac{1}{2}(1,1,-1,-1), \quad q^{3}=\frac{1}{2}(1,-1,1,-1), \quad q^{4}=\frac{1}{2}(1,-1,-1,1), \\
& q^{\dot{1}}=\frac{1}{2}(-1,1,1,1), \quad q^{\dot{2}}=\frac{1}{2}(1,-1,1,1), \quad q^{\dot{3}}=\frac{1}{2}(1,1,-1,1), \quad q^{\dot{4}}=\frac{1}{2}(1,1,1,-1) .
\end{aligned}
$$

All the coefficients are determined by considering various examples of the OPE with the free field realization. For example, to have $e^{i q_{B}^{\mathrm{i}} \phi^{B}}$ we need to consider,

$$
\begin{aligned}
& e^{i q_{B}^{1} \phi^{B}}(z) \cdot e^{-i \phi^{1}}(-z) \sim e^{i q_{B}^{\mathrm{i}} \phi^{B}}\left(1+i z \partial\left(q_{B}^{1} \phi^{B}+\phi^{1}\right)(0)+O\left(z^{2}\right)\right), \\
& e^{-i q_{B}^{2} \phi^{B}}(z) \cdot e^{i \phi^{2}}(-z) \sim e^{i q_{B}^{\mathrm{i}} \phi^{B}}\left(1+i z \partial\left(-q_{B}^{2} \phi^{B}-\phi^{2}\right)(0)+O\left(z^{2}\right)\right), \\
& e^{-i q_{B}^{3} \phi^{B}}(z) \cdot e^{i \phi^{3}}(-z) \sim e^{i q_{B}^{\mathrm{i}} \phi^{B}}\left(1+i z \partial\left(-q_{B}^{3} \phi^{B}-\phi^{3}\right)(0)+O\left(z^{2}\right)\right), \\
& e^{-i q_{B}^{4} \phi^{B}}(z) \cdot e^{i \phi^{4}}(-z) \sim e^{i q_{B}^{\mathrm{i}} \phi^{B}}\left(1+i z \partial\left(-q_{B}^{4} \phi^{B}-\phi^{4}\right)(0)+O\left(z^{2}\right)\right) .
\end{aligned}
$$

Summing over the r.h.s., we find the coefficient of $z \partial \Sigma^{\dot{\alpha}}$ also vanishes. Our goal of this appendix (C.1) follows in this way.

\section{References}

[1] A. Pankiewicz, Strings in plane wave backgrounds, Fortschr. Phys. 51 (2003) 1139 hep-th/0307027;

J.C. Plefka, Lectures on the plane-wave string/gauge theory duality, Fortschr. Phys. 52 (2004) 264 hep-th/0307101;

J.M. Maldacena, Tasi 2003 lectures on AdS/CFT, hep-th/0309246;

M. Spradlin and A. Volovich, Light-cone string field theory in a plane wave, hep-th/0310033;

D. Sadri and M.M. Sheikh-Jabbari, The plane-wave/super Yang-Mills duality,

hep-th/0310119;

M.-x. Huang, Aspects of supersymmetric gauge theory and string theory, hep-th/0404211.

[2] G. Georgiou and G. Travaglini, Fermion bmn operators, the dilatation operator of $N=4$ SYM and pp-wave string interactions, J. High Energy Phys. 04 (2004) 001 hep-th/0403188. 
[3] D.J. Gross, A. Mikhailov and R. Roiban, A calculation of the plane wave string hamiltonian from $N=4$ super-Yang-Mills theory, J. High Energy Phys. 05 (2003) 025 hep-th/0208231; J. Gomis, S. Moriyama and J.-w. Park, Sym description of SFT hamiltonian in a pp-wave background, Nucl. Phys. B 659 (2003) 179 hep-th/0210153;

J. Pearson, M. Spradlin, D. Vaman, H. Verlinde and A. Volovich, Tracing the string: bmn correspondence at finite $J^{2} / N$, J. High Energy Phys. 05 (2003) 022 hep-th/0210102.

[4] R. Roiban, M. Spradlin and A. Volovich, On light-cone SFT contact terms in a plane wave, J. High Energy Phys. 10 (2003) 055 hep-th/0211220.

[5] J. Gomis, S. Moriyama and J.-w. Park, Sym description of pp-wave string interactions: singlet sector and arbitrary impurities, Nucl. Phys. B 665 (2003) 49 hep-th/0301250.

[6] R. Dijkgraaf and L. Motl, Matrix string theory, contact terms and superstring field theory, hep-th/0309238.

[7] L. Motl, Proposals on nonperturbative superstring interactions, hep-th/9701025;

S. Sethi and L. Susskind, Rotational invariance in the m(atrix) formulation of type-IIB theory, Phys. Lett. B 400 (1997) 265 hep-th/9702101;

T. Banks and N. Seiberg, Strings from matrices, Nucl. Phys. B 497 (1997) 41 hep-th/9702187.

[8] R. Dijkgraaf, E. Verlinde and H. Verlinde, Matrix string theory, Nucl. Phys. B 500 (1997) 43 hep-th/9703030.

[9] M.B. Green and J.H. Schwarz, Superstring interactions, Nucl. Phys. B 218 (1983) 43;

M.B. Green, J.H. Schwarz and L. Brink, Superfield theory of type-II superstrings, Nucl. Phys. B 219 (1983) 437.

[10] E. Witten, Noncommutative geometry and string field theory, Nucl. Phys. B 268 (1986) 253.

[11] D.J. Gross and A. Jevicki, Operator formulation of interacting string field theory, Nucl. Phys. B 283 (1987) 1; Operator formulation of interacting string field theory, 2, Nucl. Phys. B 287 (1987) 225.

[12] A. LeClair, M.E. Peskin and C.R. Preitschopf, String field theory on the conformal plane, 1. Kinematical principles, Nucl. Phys. B 317 (1989) 411.

String field theory on the conformal plane, 2. Generalized gluing, Nucl. Phys. B 317 (1989) 464.

[13] G.T. Horowitz and A. Strominger, Translations as inner derivations and associativity anomalies in open string field theory, Phys. Lett. B 185 (1987) 45.

[14] H. Hata and S. Moriyama, Observables as twist anomaly in vacuum string field theory, J. High Energy Phys. 01 (2002) 042 hep-th/0111034;

H. Hata, S. Moriyama and S. Teraguchi, Exact results on twist anomaly, J. High Energy Phys. 02 (2002) 036 hep-th/0201177.

[15] J. Greensite and F.R. Klinkhamer, New interactions for superstrings, Nucl. Phys. B 281 (1987) 269; Contact interactions in closed superstring field theory, Nucl. Phys. B 291 (1987) 557

M.B. Green and N. Seiberg, Contact interactions in superstring theory, Nucl. Phys. B 299 (1988) 559;

J. Greensite and F.R. Klinkhamer, Superstring amplitudes and contact interactions, Nucl. Phys. B 304 (1988) 108. 
[16] I.W. Taylor, D-brane field theory on compact spaces, Phys. Lett. B 394 (1997) 283 hep-th/9611042.

[17] T. Banks, W. Fischler, S.H. Shenker and L. Susskind, M-theory as a matrix model: a conjecture, Phys. Rev. D 55 (1997) 5112 hep-th/9610043.

[18] G.E. Arutyunov and S.A. Frolov, Virasoro amplitude from the $S^{N} \mathbf{R}^{24}$ orbifold sigma model, Theor. Math. Phys. 114 (1998) 43 [hep-th/9708129]; Four graviton scattering amplitude from $S^{N} \mathbf{R}^{8}$ supersymmetric orbifold sigma model, Nucl. Phys. B 524 (1998) 159 hep-th/9712061.

[19] P. Di Francesco, P. Mathieu and D. Senechal, Conformal field theory, Springer, New York 1997. 